\title{
Lipid metabolism in the chick embryo
}

\section{By R. C. Noble, Hannah Research Institute, Ayr KA6 ${ }_{5} \mathrm{HL}$}

Once it is laid, the fertile egg provides an environment that is closed to all nutritional influences other than simple gaseous exchange. Some $5-6 \mathrm{~g}$ of the egg is lipid and is almost wholly confined to the yolk. Although doubt may be expressed about the value of the yolk lipids to human nutrition, their importance for chick embryonic development is unequivocal. Of the $21 \mathrm{~d}$ development of the chick embryo, the last $7 \mathrm{~d}$ are notable as an intense period of lipid metabolism. During the rapid embryonic growth that occurs over this time, virtually the entire lipid content of the yolk is mobilized and absorbed into the embryonic tissues (Noble \& Moore, 1964, 1966; Romanoff, 1967). The assimilation of such a large concentration of lipid over such a short period of time is characterized by extensive, and in some instances unique, lipid compositional and metabolic changes.

About $90 \%$ of the total energy requirements of the developing embryo are derived from fatty acid oxidation (Romanoff, 1967). The oxidative requirements and mechanisms of this process, which can be observed from about the 8th day of incubation, are similar to those for fatty acid oxidation in mammalian tissues (Koerker \& Fritz, 1970; Pugh \& Sidbury, 1971). With the extensive availability of lipid substrates, the extent of lipogenesis within the embryonic tissues, although detectable, remains very low up to the time of hatching (Goodridge, 1968, 1973a,b; Joshi \& Sidbury, 1976). However, circumstances can be imposed on the embryo under which the lipogenic capabilities may be stimulated before this time, indicating the potential ability of the embryonic tissues to adapt metabolically to changing substrate supply (Koerker \& Fritz, 1970; Donaldson et al. 1971; Goodridge et al. 1974). Through its role as an energy source and supply of essential tissue components, lipid transfer from the yolk to the embryo constitutes a very important part of an interrelated chain of events that ends in hatching. Over a whole range of nutritional and environmental circumstances, therefore, increased mortality has been associated with changes to the various distinctive embryonic tissue lipid patterns (Moore \& Doran, 1962; Noble \& Moore, 1966; Balnave, 1970; Singh et al. 1972; Donaldson, 1981; Kuhn \& Logan, 1983; Noble et al. 1985).

\section{The yolk lipid}

With the onset of laying in response to hormonal changes, normal plasma lipid patterns in the hen undergo considerable alterations ( $\mathrm{Yu}$ et al. 1976; Chapman, 1980). Synthesis in the liver increases considerably the overall concentrations of the plasma lipids (Table I), almost wholly through a rise in the concentration of triglyceride and very-low-density lipoprotein fractions, which are then incorporated into the developing ovarian follicles by a process of receptor-mediated 
Table I. Composition of the lipoprotein fractions in plasma of non-laying and laying hens

Lipoprotein...

Total lipid (mg/l plasma)

Triglycerides (\% total lipid in lipoprotein)

Phospholipids (\% total lipid in lipoprotein)

\begin{tabular}{|c|c|c|}
\hline \multicolumn{3}{|c|}{ Non-laying } \\
\hline VLD & LD & $\mathrm{HD}$ \\
\hline 610 & 1500 & 205 \\
\hline 64 & 25 & \\
\hline 2 I & 48 & \\
\hline
\end{tabular}

\begin{tabular}{|c|c|c|}
\hline \multicolumn{3}{|c|}{ Laying } \\
\hline VLD & LD & HD \\
\hline $1225^{\circ}$ & $125^{\circ}$ & 1000 \\
\hline 64 & 49 & 1 \\
\hline 29 & 37 & 5 \\
\hline
\end{tabular}

57

VLD, very-low-density; LD, low-density; HD, high-density.

endocytosis (Perry \& Gilbert, 1979). As a result, the lipids of the yolk consist in the main of triglycerides (7r\%), phospholipids (22\%) and free cholesterol $(6 \%)$; phosphatidyl choline and phosphatidyl ethanolamine account for about 70 and $24 \%$ respectively of the total phospholipids (Noble \& Moore, 1966). Structural analyses of the triglycerides and phospholipids in the plasma and developing ovarian follicles (Christie \& Moore, I972) support the overall view that, with the exception of early development, the lipoprotein complexes transported by the plasma from the liver are deposited intact in the ova.

\section{Removal of yolk lipid}

From about the $4^{\text {th }}$ to $5^{\text {th }}$ days of incubation, the contents of the yolk become increasingly bound by an extensive extra-embryonic membrane, the yolk-sac membrane, which is recognizable as an extension of the small intestine (Romanoff, 1960). Responsibility for the embryonic assimilation of the yolk lipids, therefore, rests with their ability to pass across this membrane into an associated network of capillaries continuous with the embryonic circulation. During the last $8 \mathrm{~d}$ of incubation, there is a preferential removal of lipid from the yolk contents amounting to about $34^{\circ} \mathrm{mg} / \mathrm{d}$ (Fig. I); between days 13 and 17 there is a considerable relocation of the yolk lipid into the yolk-sac membrane such that, by the $17^{\text {th }}$ day, the membrane contains as much lipid as the contents (Noble \& Moore, $1967 b$ ).

In contrast to lipid assimilation by the small intestine of mammals, it has now been established from both morphological and biochemical evidence that yolk lipid uptake by the membrane occurs through non-specific phagocytosis. Thus electron-microscopic studies have shown the sequential engulfment of intact lipid droplets and their subsequent appearance at the apical surface of the endodermal cells (Lambson, 1970). Although the presence of various lipolytic enzymes within the yolk has been reported (Zacks, 1954), extracellular digestion plays a neglible part in the uptake of the major lipids into the yolk-sac membrane. Almost all the analytical findings concerned with the yolk lipid contents over the incubation period are reconcilable with there being no lipid breakdown before removal (Noble $\&$ Moore, $1964,1967 b, c)$. The relative proportions of the two major yolk lipids, 


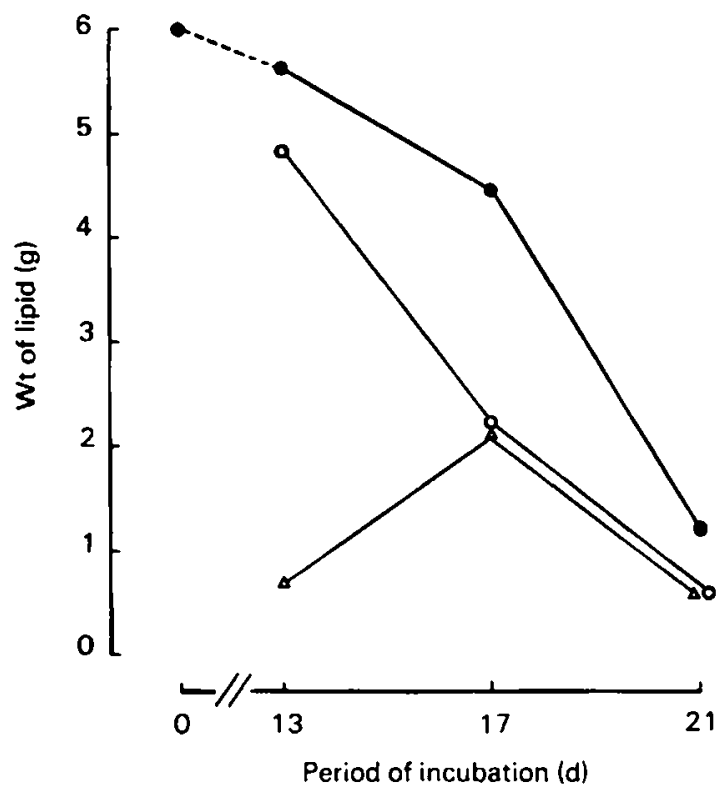

Fig. I. Mean weights of total lipid in the total yolk $(O)$, yolk contents $(O)$ and yolk-sac membrane $(\triangle)$ during development.

namely triglycerides and phospholipids, remain unchanged during the last week of incubation. Low levels only of monoglycerides, diglycerides, free fatty acids and lysophospholipids have been detected during the most intense period of yolk lipid uptake, whilst the fatty acid compositions and structures of the triglycerides and major phospholipids show little change. However, some preferential removal of lipid components does occur. Thus during the last week of incubation there is a significant increase in the proportion of yolk phosphatidyl choline relative to phosphatidyl ethanolamine, in particular through the removal of phosphatidyl ethanolamine species containing high levels of arachidonic and docosahexaenoic acids (Noble \& Moore, 1967c). Concomitant changes in the contents of more saturated phosphatidyl ethanolamine species were noted. That there is a similar preferential removal of triglycerides containing higher levels of polyunsaturated fatty acids (Isaaks et al. 1964) has not been substantiated by other investigations (Noble \& Moore, 1964, 1967b). Several changes have been observed to occur in the proportions of the various major lipoproteins within the yolk during incubation (Saito et al. 1965). The similarities observed between the relative contents, fatty acid compositions and structures of the triglycerides and phospholipids of the yolk contents and yolk-sac membrane during the last week of incubation (Noble \& Moore, $1964,1967 b, c)$ provide further evidence that there is no extensive breakdown and resynthesis during uptake into the membrane.

\section{Lipid accumulation by the embryo}

During the first $13 \mathrm{~d}$ of incubation, lipid accumulation in the embryo amounts to only about $35^{\circ} \mathrm{mg}$. Between days $\mathrm{I}_{3}$ and $\mathrm{I} 5$ a further $23^{\circ} \mathrm{mg}$ lipid are transferred 
Table 2. Compositions of the lipid (\% of total) present in the yolk contents and yolk-sac membrane at different stages of incubation (Noble \& Moore, 1967b)

Period of incubation (d)
Triglycerides
Free cholesterol
Esterified cholesterol
Phospholipids

\begin{tabular}{rrr}
\multicolumn{3}{c}{ Yolk contents } \\
\multicolumn{1}{c}{13} & \multicolumn{1}{c}{17} & \multicolumn{1}{c}{21} \\
71.8 & 72.0 & 75.9 \\
$6 \cdot 1$ & 6.0 & 5.0 \\
0.4 & 0.5 & 0.5 \\
21.4 & 21.1 & 18.2
\end{tabular}

\begin{tabular}{|c|c|c|}
\hline \multicolumn{3}{|c|}{ Yolk-sac membrane } \\
\hline 13 & 17 & 21 \\
\hline $7^{6 \cdot 9}$ & $71 \cdot 9$ & $68 \cdot 6$ \\
\hline I.9 & $2 \cdot 1$ & $2 \cdot 3$ \\
\hline $3 \cdot 3$ & 4.0 & 6.9 \\
\hline 15.7 & 19.4 & 17.5 \\
\hline
\end{tabular}

from the yolk to the embryo but between day 15 and day 21 the overall uptake of lipid is increased considerably such that, during the last $2 \mathrm{~d}$ of incubation, the yolk lipids are removed at a rate of over I $\mathrm{g} / \mathrm{d}$ (Noble \& Moore, 1964); this is accompanied by marked increases in the amounts of lipid in embryonic tissues. Morphological and biochemical evidence indicates that some hydrolysis and re-esterification of the triglycerides and phospholipids, together with re-assembly into newly-synthesized lipoproteins, occurs within the yolk-sac membrane before their passage into the embryo. Lipase activity within the yolk-sac membrane and a sequence of lipid droplet elaborations within the individual cells, in a manner resembling systems known to involve hydrolysis and re-esterification, have both been observed (Zacks, 1954; Lambson, 1970). Distinct differences exist between the structures and lipid contents of the major lipoprotein classes of the yolk and embryonic plasma (Schjeide, 1963). When compared with the yolk contents, a feature of the yolk-sac-membrane lipids is its markedly higher esterified cholesterol :free cholesterol value (Table 2) and the very much higher concentration of oleic acid in the cholesteryl esters (Noble \& Moore, ${ }_{1967} 6$ ); the synthesis of these cholesteryl esters by an acyl-coenzyme A-cholesterol acyltransferase (ACAT) enzyme system in the yolk-sac membrane has now been shown (Noble et al. 1984).

\section{Comparative tissue studies}

The most unusual feature of the tissue lipid changes that occur in the embryo during development is that displayed by the liver. Although there is a large accumulation of lipid within the liver during yolk assimilation, by day ig of incubation it comprises about $5 \%$ of the embryonic total: its composition is quite unusual and bears no resemblance to the lipid absorbed from the yolk. The lipids of the liver exhibit very high levels of cholesteryl esters (Table 3) with a very high content ( $75-80 \%$ of total fatty acids) of oleic acid (Moore \& Duran, 1962; Noble \& Moore, 1964, 1966). Comparison with the lipid compositions of the other embryonic tissues has shown that this accumulation of cholesteryl oleate is confined to the liver. The extrahepatic tissues display low concentrations only of cholesteryl esters in which oleic acid accounts for no more than $40-50 \%$ of the total long-chain fatty acids present. The cholesteryl esters of the yolk contents, which comprise less than $1 \%$ of the total lipid present (Noble \& Moore, 1967a), also do not contain high levels of oleic acid. It is doubtful therefore that the 
Table 3. Changes in total lipid content, cholesteryl ester content and its oleic acid concentration in the embryonic liver during development (Noble \& Moore, I964)

$\begin{array}{lccc}\text { Period of incubation (d) } & 15 & 17 & 19 \\ \text { Lipid (mg/liver) } & 24 & 60 & 150 \\ \text { Cholesteryl esters (\%): } & & & \\ \quad \text { In dry tissue } & 8 \cdot 7 & 13 \cdot 3 & 31 \cdot 5 \\ \quad \text { In total lipid } & 33 \cdot 9 & 50 \cdot 0 & 70 \cdot 2 \\ \text { Oleic acid in cholesteryl esters (\%) } & 76 \cdot 8 & 77 \cdot 3 & 78 \cdot 4\end{array}$

assimilation of the yolk lipid is directly responsible for the cholesteryl ester accumulation in the liver. However, the increasing presence in the yolk-sac membrane of cholesteryl esters displaying levels of oleic acid similar to those of the liver during the period of intense yolk lipid assimilation (Noble \& Moore, 1967b) suggested the yolk-sac membrane as the major hepatic source. Comparative investigations of the cholesterol-esterifying systems present in the yolk-sac membrane and liver have provided further evidence for this probability (Noble et al. 1984); although the liver possessed cholesterol esterifying ability, its specificity for oleic acid was very much lower than the cholesterol esterification in the yolk-sac membrane (Table 4 ).

Cholesterol for esterification is readily available from assimilation of the yolk contents; indeed, other than the brain where cholesterol synthesis is very active and accounts for about $90 \%$ of the total cholesterol content, the yolk provides almost all the cholesterol which accumulates in the embryonic tissues (Connor et al. 1969). The observation (Noble \& Moore, 1964) that the accumulation of cholesteryl oleate in the liver during the last week of incubation was inversely related to the decrease in the total yolk lipid concentration had prompted the suggestion that the cholesteryl esters which accumulate in the liver might have played a specific role in lipid transport and assimilation from the yolk. The demonstration that the yolk-sac membrane, which is the major site of lipoprotein synthesis and assembly before uptake by the embryo, was also the main site for cholesteryl ester synthesis would support such a suggestion. Indeed, relatively high concentrations of cholesteryl esters have been found in the lipids of the chylomicron and the very-low-density lipoprotein fractions isolated from embryonic plasma (Schjeide, 1963), indicating that the cholesteryl esters

Table 4. Oleic acid contents of cholesteryl esters synthesized in vitro or accumulated during development in the yolk-sac membrane and liver at day 15 of development (Noble et al. 1984)

$\begin{array}{lcc} & \begin{array}{c}\text { Oleic acid in } \\ \text { cholesteryl esters } \\ \text { synthesized in vitro (\%) }\end{array} & \begin{array}{c}\text { Oleic acid in } \\ \text { cholesteryl esters accumulated } \\ \text { during embryonic development (\%) }\end{array} \\ \text { Yolk-sac membrane } & 70 \cdot 2 & 71 \cdot 9 \\ \text { Liver } & 48.4 & 78.4\end{array}$


synthesized in the yolk-sac membrane are important in the assembly of the lipoprotein complexes required for lipid transport.

In accordance with its possible role in yolk-lipid uptake, the ability of the yolk-sac membrane to increase its synthesis of cholesteryl esters, in particular cholesteryl oleate, up to the $15^{\text {th }}-16$ th days of incubation coincides with the period of intensive redistribution of lipid from the yolk contents into the membrane. Furthermore, it was shown that, whereas between day 13 and day 17 the proportions of oleic acid within the cholesteryl esters of the yolk-sac membrane increased significantly, there were no further changes during the last $4 \mathrm{~d}$ of incubation. The increasing ability of the liver during incubation in vitro to synthesize cholesteryl esters containing a more conventional spectrum of fatty acids (Noble et al. 1984) accords with the finding that, following hatching, there is a rapid depletion of the cholesteryl oleate which accumulated during development. Although there is no definitive evidence for any mechanism by which the embryo may reduce the hepatic accumulation of cholesteryl esters, excretion through the bile into the small intestine might be one possible pathway. Recent investigations (Noble \& Connor, 1984) have shown that, chick embryo gall bladder compared with other animal species bile displays a very unusual lipid pattern which includes substantial quantities of cholesteryl esters (Table 5). An increase in esterified cholesterol:free cholesterol in the yolk contents, together with an increased level of oleic acid in the cholesteryl esters of the yolk contents as incubation proceeds, has also been noted (Noble \& Moore, $1967 b$ ).

Comparative studies of the fatty acid compositions of phosphatidyl ethanolamine, phosphatidyl serine, phosphatidyl choline and diphosphatidyl glycerol within the yolk, yolk-sac membrane, liver and extrahepatic tissues (Noble \& Moore, 1965, 1967a,c; Wood, 1974; Abad et al. 1976; Nakagawa et al. 1982) are consistent with extensive degradation of yolk phospholipids following assimilation and resynthesis into products which are either utilized for embryonic development or oxidized to furnish energy (Hevesy et al. 1938; Budowski et al. 1961). Sphingomyelin would appear to be absorbed from the yolk and deposited in the tissues with the minimum breakdown and resynthesis. Phosphatidyl inositol, which is absent from the yolk, is synthesized by the embryo. Although it has been suggested that yolk triglycerides are retained by the embryo without extensive degradation and resynthesis (Budowski et al. 196r), some unusual aspects of their

Table 5. Compositions of the lipid (\% of total) present in gall bladder bile of chick embryo and rabbit (Noble $\mathcal{E}^{\circ}$ Connor, 1984)

$\begin{array}{lccccc}\text { Cholesteryl } & \text { Triglycerides } & \begin{array}{c}\text { Free } \\ \text { esters }\end{array} & \begin{array}{c}\text { Oleic acid in } \\ \text { cholesterol }\end{array} & \begin{array}{c}\text { Phospholipids } \\ \text { cholesteryl } \\ \text { esters (\%) }\end{array} \\ \text { Chick embryo } & 29.6 & 12.1 & 18.1 & 40.3 & 75.3 \\ \text { Rabbit } & <0.5 & <0.5 & 12.3 & 87.5 & \text { NR }\end{array}$

NR, no result. 
fatty acid composition within the embryo, in particular a substantial content of docosahexaenoic acid, would indicate synthesis of entirely new triglyceride species during incubation.

\section{Metabolism of unsaturated fatty acids}

A further feature of the lipid metabolism of the embryonic liver is that the phospholipids, which account for some $10 \%$ of the total lipid present, exhibit unusually high contents of arachidonic acid ( $>20 \%$ of the total fatty acids), particularly during the early part of the yolk lipid mobilization (Noble \& Moore, 1967a). By contrast, arachidonic acid comprises only about $3 \%$ of the total fatty acids in the phospholipids of the yolk contents (Noble \& Moore, 1965, 1967b). Recent investigations (Noble \& Shand, 1985) have correlated the activities of the $\triangle 6$-long-chain fatty acid desaturase system (responsible for the conversion of linoleic acid to arachidonic acid) with the levels of linoleic and arachidonic acids in the yolk contents, yolk-sac membrane and liver during the last week of development and have shown that the high levels of arachidonic acid in the liver probably arise following synthesis within the yolk-sac membrane (Table 6). With the approach of hatching and the functional regression of the yolk-sac membrane, this role is taken over by the embryonic tissues themselves. Similarly, the presence in the yolk-sac membrane of an active $\Delta 9$-long-chain fatty acid desaturase system (responsible for the conversion of stearic acid to oleic acid) may also make a contribution to the oleic acid requirement of the developing embryo.

The triglycerides of the liver in particular, and extrahepatic tissues also, display surprisingly high levels of docosahexaenoic acid; in the liver the levels are considerably higher than those in the phospholipids (Noble \& Moore, 1964). The source of the docosahexaenoic acid appears to be the yolk phospholipids through a preferential absorption of phosphatidyl ethanolamine species rich in docosahexaenoic acid (Noble \& Moore, $1965,1967 b, c$ ).

The possibility exists therefore of a specific requirement during embryonic development for a range of polyunsaturated fatty acids which cannot be adequately supplied through the more saturated fatty acid composition of the yolk lipids. However, in the case of docosahexaenoic acid it is strange that, following preferential absorption from the yolk, the acid should be utilized in substantial amounts merely for the synthesis of liver triglycerides.

Table 6. $\Delta 6$ - and $\Delta 9$-desaturase activities of the liver and yolk-sac membrane during the last week of development (Noble \& Shand, 1985)

$\begin{array}{lcccccc}\text { Period of incubation (d) } & \overbrace{15} & 17 & 19 & \overbrace{15} & 17 & 19 \\ \begin{array}{l}\text { Linoleic desaturation } \\ \text { (pmol/min per mg protein) }\end{array} & 9.3 & 11.6 & 13.1 & 23.4 & 5.1 & 6.6 \\ \begin{array}{c}\text { Stearic desaturation } \\ \text { (pmol/min per mg protein) }\end{array} & 51.4 & 16.1 & 17.4 & 32.9 & 26.2 & 14.3\end{array}$




\section{Parental age and lipid metabolism}

Several studies have shown that parental age has a considerable effect on hatchability and early chick survival (Smith \& Bohren, 1975; Shanawany, 1985). This has proved to be a particular problem in the broiler industry where, through pressure to maintain the chick yield from each hen, hatching eggs are being taken at progressively earlier stages of the hen's life. The reduced hatchability displayed by the eggs from the very young breeder hen is associated with an abnormal distribution of overall embryonic weight (Shanawany, I985). In a study of fertile eggs from 25- and 4I-week-old broiler birds at days 15 and 19 of incubation, significant differences existed between the parental groups in the distribution and composition of lipid in the yolk contents, yolk-sac membrane and embryonic tissues (Noble et al. 1985). The amount of lipid associated with the total yolk, i.e. yolk content plus yolk-sac membrane, of the embryos from the 25-week-old parents was greater than that of the embryos from the 4I-week-old parents. The distribution of lipid between the yolk contents and yolk-sac membrane was such that in the embryos from the 25-week-old parents there was a much higher proportion of lipid still remaining in association with the yolk contents. Differences too were observed between the relative contents of major lipid constituents within the yolk and embryonic tissues. The higher mortality of the chick embryos from the very young parents was therefore associated with a malfunction of yolk lipid assimilation through a reduction in the ability to mobilize the stored lipid from the yolk contents into the yolk-sac membrane. As a result, access by the embryo to the major nutrient associated with development during the last week of incubation was being denied.

\section{Conclusions}

The transfer of lipid from yolk to embryo during the last week of development of the chick has been shown to be associated with some notable changes in the lipid compositions of the various tissues. Investigations into the accumulation and synthesis of some of the major lipid fractions within the yolk and embryo have enabled metabolic interpretations to be made of several of these changes. A central role for the yolk-sac membrane in these major lipid changes is suggested. Distinctive alterations to the lipid patterns associated with normal embryonic development may enable the causative factors of reduced hatchability to be discerned.

\section{REFERENCES}

Abad, C., Bosch, M. A., Municio, A. M. \& Ribera, A. (1976). Biochimica et Biophysica Acta 431, 62-74.

Balnave, D. (1970). World's Poultry Science fournal 26, 442-460.

Budowski, P., Bottino, N. R. \& Reiser, R. (1961). Archives of Biochemistry and Biophysics 93, $483-490$.

Chapman, M. J. (1980). Journal of Lipid Research $21,789-853$.

Christie, W. W. \& Moore, J. H. (1972). Comparative Biochemistry and Physiology 4rB, 287-295. 
Connor, W. E., Johnston, R. \& Lin, D. S. (1969). Fournal of Lipid Research 10, 388-394.

Donaldson, W. E. (1981). Poultry Science 60, 1964-1970.

Donaldson, W. E., Mueller, N. S. \& Mason, J. V. (197I). Biochimica et Biophysica Acta 248, 34-40.

Goodridge, A. G. (1968). Biochemical foumal 108, 655-661.

Goodridge, A. G. (1973a). Fournal of Biological Chemistry 248, 1924-1 931 .

Goodridge, A. G. (1973b). Foumal of Biological Chemistry 248, 1939-1945.

Goodridge, A. G., Garay, A. \& Silpananta, P. (1974). Fournal of Biological Chemistry 249, I469-1475.

Hevesy, G., Levi, H. B. \& Rebbe, O. H. (1938). Biochemical Fournal 32, $2147^{-2}$ I 55.

Isaaks, R. E., Davies, R. E., Ferguson, T. M., Reiser, R. \& Couch, J. R. (1964). Poultry Science 43, $113-120$.

Joshi, V. C. \& Sidbury, J. B. Jr. (1976). Archives of Biochemistry and Biophysics 173, 403-414.

Koerker, D. J. \& Fritz, I. B. (1970). Canadian Fournal of Biochemistry 48, $418-424$.

Kuhn, D. E. \& Logan, D. M. (1983). Canadian fournal of Biochemistry and Cell Biology 6r, $378-386$.

Lambson, R. O. (1970). American fournal of Anatomy $129,1-20$.

Moore, J. H. \& Doran, B. M. (1962). Biochemical fournal 84, 506-513.

Nakagawa, Y., Waku, K. \& Ishima, Y. (1982). Biochimica et Biophysica Acta 712, 667-676.

Noble, R. C. \& Connor, K. (1984). Lipids 19, 64-67.

Noble, R. C., Connor, K. \& Smith, W. K. (1984). Poultry Science 63, 558-564.

Noble, R. C., Lonsdale, F., Connor, K. \& Brown, D. (1985). Poultry Science (In the Press).

Noble, R. C. \& Moore, J. H. (1964). Canadian fournal of Biochemistry 42, 1729-1 74I.

Noble, R. C. \& Moore, J. H. (1965). Canadian Journal of Biochemistry 43, 1677-1686.

Noble, R. C. \& Moore, J. H. (1966). In Physiology of the Domestic Fouol, pp. 86-ror [C. Horton-Smith and E. C. Amoroso, editors]. Edinburgh: Oliver and Boyd Ltd.

Noble, R. C. \& Moore, J. H. (1967a). Canadian fournal of Biochemistry 45, 627-639.

Noble, R. C. \& Moore, J. H. (1967b). Canadian Fournal of Biochemistry 45, 949-958.

Noble, R. C. \& Moore, J. H. (rg67c). Canadian fournal of Biochemistry 45, I $125-1133$.

Noble, R. C. \& Shand, J. H. (1985). Lipids 20, 278-282.

Perry, M. M. \& Gilbert, A. B. (1979). Foumal of Cell Science 39, 257-272.

Pugh, E. \& Sidbury, J. B. Jr. (197I). Biochimica et Biophysica Acta 239, 376-383.

Romanoff, A. L. (1960). The Avian Embryo. New York: Macmillan.

Romanoff, A. L. (1967). Biochemistry of the Avian Embryo. New York: Macmillan.

Saito, Z., Martin, W. G. \& Cook, W. H. (1965). Canadian Fournal of Biochemistry 43, $1755^{-1} 770$.

Schjeide, O. A. (1963). In Progress in the Chemistry of Fats and Other Lipids, vol. 5, pp. 253-290 [R. T. Holman, W. O. Lundberg and T. Malkin, editors]. Oxford: Pergamon Press.

Shanawany, M. M. (1985). British Poultry Science 25, 449-455.

Singh, R. A., Weiss, J. F. \& Naber, E. C. (1972). Poultry Science 51, 449-457.

Smith, K. P. \& Bohren, B. B. (1975). Poultry Science 54, 959-963.

Wood, R. (r974). Lipids 9, 429-439.

Yu, J. Y. L., Campbell, L. D. \& Marquardt, R. R. (1976). Poultry Science 55, 1626-1631.

Zacks, S. I. (1954). Anatomical Record 118, 509-537. 